\title{
"Near-Death Experiences with Hallucinatory Features" Defended
}

\author{
Keith Augustine, M.A. \\ Internet Infidels, Colorado Springs, $\mathrm{CO}$
}

\begin{abstract}
Three preceding commentaries present even-handed and thoughtful criticisms of "Near-Death Experiences with Hallucinatory Features." The commentators and I agree that near-death experience (NDE) reports are sometimes open to multiple interpretations, that different kinds of NDEs should be distinguished according to their diverse physiological mechanisms, and that transformations following NDEs but not other hallucinatory experiences require special explanation if NDEs are hallucinations. In this reply I discuss the most significant areas of continuing disagreement.
\end{abstract}

KEY WORDS: false perceptions; bilocation; ad hoc hypotheses; diversity of NDEs; NDE transformations.

I greatly appreciate the even-handed and thoughtful commentaries on "Near-Death Experiences with Hallucinatory Features" provided by Janice Holden, Peter Fenwick, and William Serdahely. Since the commentators often gave insightful comments, I will largely restrict my response to the most significant areas of disagreement.

Holden's first main point is that anecdotes are sometimes open to multiple interpretations. I concur that her possible alternative interpretation of the report of an NDE during an aerial attack in World War II relies on an "equally plausible sequence of events" as the interpretation that the NDE contained out-of-body discrepancies. However, this approach can go only so far: alternative interpretations

Keith Augustine, M.A., is Executive Director and Scholarly Paper Editor of Internet Infidels, a nonprofit educational organization dedicated to defending and promoting metaphysical naturalism on the Internet. Reprint requests should be addressed to $\mathrm{Mr}$. Augustine at Internet Infidels, PO Box 142, Colorado Springs, CO 80901-0142; e-mail: kaugust@infidels.org. 
may account for some potentially hallucinatory experiences without requiring one to see them as hallucinations, but will undoubtedly not account for all of them. For example, in Robert Crookall's Case 731 the subject explicitly reported seeing bars on his bedroom window during an OBE that did not exist and that "prevented" him from leaving the room (1972, pp. 89-90).

Holden's second main point is that failure to note a feature of the physical environment during an out-of-body NDE provides evidence of selective attention to detail, not of hallucination. She asks "whether incomplete visual perception of the entire 'available' scene during an OBE necessarily justifies a conclusion that the experience was hallucinatory." Again, while selective attention may account for some perceptual omissions, it cannot plausibly account for all of them. She notes, for instance, Margot Grey's observation of NDErs who reported wanting to see their physical bodies during their out-of-body NDEs but nevertheless failing to do so. Similarly, Oliver Fox reported that, in the majority of his induced OBEs, he could not see his normal physical body lying on the bed despite specifically looking for it there (Fox, 1962).

Though Holden writes that 'preoccupation fits with many NDErs' descriptions of their selective, and sometimes exclusionary, perception in the out-of-body state," in fact it accounts only for selective attention. Those who not only wanted to see their bodies during their OBEs but were actively looking for them could hardly be said to be victims of selective attention to detail or preoccupation with other things. No obstacle, physical or mental, was blocking their perception. Exclusionary imagery, then, is better explained in terms of hallucination than veridical perception of selective details. Indeed, by failing to offer any conceivable explanation for exclusionary perception, Holden implicitly concedes that there is none other than hallucination.

Both of Holden's main points, then, fail to show that we cannot be justifiably confident that genuine discrepancies between NDE content and consensual reality exist. More striking examples are instances of false perception: "perceiving" objects during OBEs that are not there, such as Crookall's report of seeing nonexistent bars on a bedroom window. How does Holden explain cases containing invented objects as anything other than reports of hallucinations? Just what sorts of perceptual errors would, according to Holden, render a given NDE a hallucination?

Next Holden considers the significance of bodily sensations incorporated into NDE content. She writes that the assumption "that 
consciousness is always either in or out of the body" may be true of "phenomena of consciousness in the gross physical world," but is not necessarily true of "phenomena of consciousness that may transcend that world," just as the assumption that physical objects have a definite location seems true of "superatomic" phenomena but not of subatomic particles in a superposed state. To say that any aspects of the human mind "transcend" the gross physical world is to say that they somehow extend outside of it, to elsewhere. But that another world may function according to different laws does not really help, as the bare possibility that one's astral duplicate can simultaneously "occupy" the physical body and travel elsewhere is not an argument for the plausibility of such bilocation. Alternatively, perhaps NDErs' physical bodies are like voodoo dolls whose manipulation can affect their astral duplicates no matter where their ethereal doubles are located.

Similarly, Holden proposes that encounters with living persons are explicable by the alternative hypothesis that NDEs "may take a form that the ... [NDEr] is most likely to recognize and accept." Instead of granting that living persons are seen more often than deceased ones in childhood NDEs simply because children tend to know far fewer deceased persons than living ones, she hypothesizes that "a transcendent benevolent entity seeking to encourage ongoing physical existence might most effectively appear in the form of a living person known to the NDEr." This is quite an extravagant alternative. Instead, perhaps some NDEs start out as hallucinations and then, like Pinocchio, somehow "become" real. That NDEs are hallucinations whose content is derived from NDErs' imaginations in order to fulfill a psychological need in the face of a threat to well-being is a simpler and far more plausible explanation than that of impersonation by purely hypothetical celestial beings. We have far better evidence that hallucinatory altered states occur than we have that any "transcendent entities" exist.

Furthermore, a transcendent entity could be said to take any form imaginable, including those of fictional characters and mythological creatures. Similar points apply to alternative explanations for unfulfilled prophetic NDEs: while it is possible that transcendent entities offered NDErs false visions of the future, or that humanity changed its ways just in time to avert disaster, such responses are hackneyed and inane compared to the most likely explanation for false NDE prophecies.

Bodily sensations and encounters with living persons in NDEs are unquestionably compatible with "the possibility that a living individ- 
ual's consciousness may function in more than one 'place' at the same time and usually unbeknownst to that person." But if we allow this hypothesis into our pool of live options, why stop there? Perhaps the characters in our dreams are "unaware bilocations" of friends who may be shopping at the time, or transcendent impersonators; this is no less consistent with the actual content of our dreams. Shall we pretend that these explanations are really on a par with the hypothesis that dream characters are nothing more than a product of the dreamer's imagination? If not, then similar counterexplanations for NDEs with ostensibly hallucinatory features are equally implausible relative to the chief explanation for them. To suggest that no conceivable evidence could "provide sufficient weight to favor strongly one of these hypotheses over the other" is analogous to maintaining that the theories that crop circles are formed by spirit activity, extraterrestrial intervention, or meteorological vortices are as plausible as the hypothesis that they are created by human activity.

These counterexplanations seem entirely ad hoc: there appears to be no independent reason to postulate something as exotic as bilocation to account for bodily sensations or encounters with living persons in NDEs other than to save a survivalist interpretation of NDEs. Without some positive reason for invoking them apart from accounting for such NDEs in survivalist terms, they appear simply to explain away otherwise contradictory evidence. Clearly a survivalist interpretation can be amended with any number of ad hoc modifications when confronted with ostensibly inconsistent evidence; but such evidence does, on the face of it, undermine that interpretation. That NDEs are hallucinations explains apparent discrepancies between NDE content and consensual reality more simply than, and much better than, a survivalist interpretation qualified with various ad hoc amendments. Ad hoc explanations simply supply a way to dismiss evidence inconsistent with one's theories.

Finally, these alternatives make any inferences about the nature of NDEs given their content unfalsifiable, making the conclusion that any particular NDE is a hallucination given its content unreachable; for any possible discrepancy between NDE content and consensual reality can be explained away in this manner. One might as well hypothesize that the beings encountered in "bad LSD trips" are transcendent malevolent entities seeking to increase psychonauts' anxiety most effectively by appearing in a maximally frightening form. Moreover, these hypothesis-saving devices cut both ways: An 
entrenched hallucination theorist could just as easily explain away reproducible experimental evidence of veridical paranormal perception among certain OBE adepts as fantastically unlikely coincidence, but coincidence nonetheless. The point of introducing these sorts of evidence is to test our interpretations against the actual data that have been collected. Ad hoc counterexplanations undermine that aim effectively by treating the data as irrelevant. If such drastic measures are acceptable, then what is the point of doing research in the first place?

When Holden writes that "just as citing only cases involving no perceptual errors can give a false impression of the overall picture of perceptual accuracy during near-death OBEs, so can citing only those involving errors," she misunderstands the purpose of my survey. It was never my intention to suggest that NDEs with overtly hallucinatory features are at all typical or even common among NDE reports. I never overstated their frequency, characterizing them as occasional at best, and noting, for example, that only 14 percent of the Fenwicks' cases included encounters with living persons.

Holden rightly characterizes my selection of cases as "highly selective and exclusive," but that is irrelevant. What is problematic for a survivalist interpretation is not the frequency of such NDEs, but that they occur at all. Serdahely makes the same mistake when he complains that I inappropriately generalized from "the exception or the rare or infrequent finding" in making my case, noting my inclusion of an Elvis sighting in an NDE and writing: "A reporting of Elvis is extremely rare in the NDE literature." Serdahely seems to think that if such instances are rare enough, they provide no grounds for doubting a survivalist interpretation of most NDEs. But this is like arguing that the reliability of regressed memories of Satanic ritual abuse is not undermined when only a small minority of cases include demonstrably false memories of the disposal of human remains. For any analogous phenomenon, it is virtually assured that only a small number of cases will be detailed enough to reveal truth-claims that can subsequently be tested against consensual reality. Such specificity in anecdotes is almost inherently rare, and consequently the discovery of discrepancies between reports and consensual reality will be at least as rare as those anecdotes themselves.

For the sake of argument, let us presume that Holden's survey of the professed accuracy of NDE details compared against consensual reality in published accounts is representative of all published 
accounts, all reports of NDEs whether they are published or not, and even all NDEs whether they are reported to anyone else or not. In that case, I am happy to concede that only 8 percent of NDEs contain discrepancies between NDE content and consensual reality. But I fail to see the significance of such a finding. Only 8 percent of prototypical Western NDEs include a barrier or border between life and death (van Lommel, van Wees, Meyers, and Elfferich, 2001), but NDE researchers do not regard this element as insignificant because of its infrequency.

If something leaves the body and perceives the physical world during out-of-body NDEs, and occasionally travels into some kind of objective afterlife environment, then on the face of it discrepancies between NDE content and consensual reality should not occur. Citing her own survey as confirmation, Holden concedes that "some NDEs contain perceptual errors," but adds that such errors "do not justify the conclusion that all of those experiences were hallucinatory." Certainly not all such errors are indicative of hallucination, but most of them are best explained in those terms. Overall, Holden's counterexplanations of out-of-body discrepancies, bodily sensations, and encounters with living persons - to say nothing of more explicitly hallucinatory imagery - are logically possible, but inadequate as explanatory hypotheses.

Finally, Holden writes that even if some NDEs are hallucinations, "logic prevents the conclusion that all NDEs are necessarily hallucinatory." Undoubtedly, the existence of hallucinatory NDEs does not prove that all NDEs are hallucinations. But NDEs with overt hallucinatory features do give us some grounds to suspect that NDEs that are not so explicitly hallucinatory are hallucinations as well. Suppose that a certain percentage of "alien abductees," whose experiences are typical of those of other alien abductees in all other respects, report being abducted in very public places, contrary to the consensual reality established by innumerable other observers. If we set aside potential ad hoc counterexplanations, such as that aliens can blank out the memories of all else who are present, we can quite reasonably conclude that, at least for this percentage of abductees, alien abduction experiences are imaginary. But the discovery of otherwise indistinguishable discrepant alien abduction experiences would cast serious doubt about extraterrestrial visitation being the cause of alien abduction experiences, especially in light of our background knowledge that there has never been a single indisputable instance of extraterrestrial visitation. Would not all alien abduction 
experiences be better explained on more mundane psychological hypotheses under these circumstances?

Fenwick objects that trying to classify private experiences as either "brain-based hallucinations, or a peek into the afterlife" seems "doomed from the outset" because we cannot conclusively distinguish between the two: neither categorization is falsifiable. In principle, one could construct a concept of the afterlife so broad that any conceivable private experience could be construed as a vision of an afterlife. For instance, a disembodied mind might generate a solipsistic environment that could very well consist of entirely illusory features: in effect a brain-free hallucination. Conversely, one could define hallucinations so broadly that every human experience could be construed as a hallucination. But despite these caveats, we routinely diagnose certain experiences as hallucinations and others as veridical. Ostensible "afterlife experiences" may not be directly testable insofar as we have no reliable "roadmap" of an afterlife to check against, but this does not mean that the content of such experiences cannot offer us strong grounds from which to infer their true nature.

Next Fenwick criticizes my paper for failing to address the causes of NDEs. I largely sidestepped the issue here because the causes of NDEs are not yet known, only speculated upon. He also criticizes me, rightly, for treating NDEs as if they were a single phenomenon, but I might be forgiven for unreflectively following the apparent milieu of near-death studies on this point. It seems to me that the vast majority near-death researchers have been treating NDEs as a single phenomenon in the sense that Fenwick outlines, rather than as a simplifying label for a variety of different experiences. Researchers have certainly acknowledged that prototypical Western NDEs are experientially quite different from the occasional "hellish" NDEs or "meaningless void" experiences, and that some NDEs mix elements of these different experiential types. But to my knowledge only one study has sought to distinguish types of NDEs systematically in terms of their undeniably diverse physiological triggers (Twemlow, Gabbard, and Coyne, 1982). I am no less guilty of this shortcoming, and am grateful that Fenwick has brought it to our attention.

Fenwick lists some of the physiologically diverse conditions precipitating NDEs, arguing that a neurophysiological mechanism common to all of them is highly unlikely, but that "there may be several mechanisms that lead to a common experience." He adds that I treat OBEs and NDEs "in different physiological states as if they all 
had the same physiological mechanism," but that the literature reveals that "there is no disruption of normal neurophysiological functioning [in spontaneous OBEs], and that they are probably dissociative states in which the experiencer will gain no veridical perception away from the body." Finally, he reiterates that common spontaneous OBEs are distinct from those accompanying sleep paralysis, rapid eye movement (REM) sleep, electrical stimulation of the temporal lobe, epileptic seizures, and cardiac arrest. (It is notable that several of the clearly hallucinatory cases I cited were cardiac arrest NDEs.) Ultimately, he suggests that prospective trials in which researchers "have some control over the psychological and physical variables" might reveal "a common [transcendental] reality that is accessed by different [neurophysiological] routes," but it is unclear what variables would need to be controlled.

In fact, my paper is silent about psychophysiological mechanisms because they are both speculative and secondary to my central point. But for the record, it is undoubtedly true that there is no one mechanism for generating NDEs; I have already noted that fear alone, absent any clear physiological trigger, can generate NDEs (Augustine, 2007). Clearly, the illusion of perceiving from somewhere outside of the body can be generated by a variety of different physiological mechanisms. Harvey Irwin (1985, 2000) has addressed the variability of OBE triggers, hypothesizing that what is physiologically crucial is extremes in arousal - either very high (for example, a near-death crisis) or very low (for example, meditation) states of cortical arousal -combined with psychological dispositions like high capacity for absorption and strong need for absorbing experiences.

According to Serdahely, I am guilty of a major oversight in maintaining that NDEs are brain-generated hallucinations without ever defining the term "hallucination." I took it for granted that the term would be widely understood without admitting of significantly different possible meanings, just as near-death researchers routinely do when using terms like "veridical perception." I think that most definitions are, for the purposes of my paper, essentially equivalent. Nevertheless, I am happy to offer a definition here: A hallucination is a sensory perception of an object, entity, or environment that does not exist outside of the mind of the percipient. In short, a hallucination is a nonveridical sensory perception.

Serdahely also takes issue with my "binary thinking" about the ontological status of NDEs, but I am unconvinced that it is 
problematic. Researchers obviously can introduce any number of complicated alternatives to either of the two most straightforward interpretations of NDEs: that NDEs are purely internal mental models, or that they are perceptions of an external environment by discarnate persons. But the additional complexity that such "middle ways" bring to the question does not, it seems to me, provide any explanatory advantages over the more straightforward explanations, and these alternatives often bring in disadvantageous conceptual problems. Consider so-called "field theories" of survival, in which the "mind field" always extends beyond the body but we only become aware of this during OBEs. Irwin asks: "is the mind field distributed uniformly throughout the cosmos or is it more 'dense' in the vicinity of the physical body? Does the mind field 'expand' to achieve access to very distant locations or does its nonphysical status imply that the field perpetually encompasses all locations?" (Irwin, 1985, p. 258). Without strong empirical grounds for taking such hazy alternatives seriously, we have no reason to treat them as accurate representations of how consciousness operates.

Like Holden, Serdahely offers alternative interpretations of some of the cases I cited. First, he points out that some ostensible NDEs with hallucinatory features may not be NDEs at all. This is a fair point, which I anticipated, since for most of the published retrospective accounts there was no evidence that researchers administered the NDE Scale (Greyson, 1983) in order to identify NDEs. So my limited criteria for identifying NDEs were whether near-death researchers categorized an experience as such, whether the accounts included experiential elements of the prototypical Western NDE, and whether the experiences were precipitated by some perceived or actual threat of harm. Nevertheless, I tried to allay the possibility of misidentification by including as many different clear NDEs with hallucinatory features as I could find. Consequently, potential misidentification of experiences that are not NDEs could account only for a small number of the cases I cite. The same could be said of the possibility that some NDE reports are entirely fabricated.

Serdahely's objection that, if NDEs are hallucinations, we should see "many more varied and diverse variations [in NDE content] similar to the nearly infinite number of idiosyncratic features reported in dreams" is also a fair point. This is a topic I will take up in the next issue of this Journal, where I acknowledge that prototypical Western NDEs are much more stereotyped than dreams. Exactly why this is so 
is unclear at the moment, but one possible explanation is that similar physiological mechanisms produce similar experiences in different individuals given their similar "hard-wiring," though that seems hard to reconcile with extant crosscultural data on NDEs. Another is that there are common sociocultural stereotypes about what is to be expected at or following death, and that these expectations shape the content of NDEs. I will address this issue later, but suffice it to say that further research is needed to answer this question confidently.

Regarding my discussion of the Fenwicks' discovery that their sample of near-death life reviews consisted of almost exclusively random memories, Serdahely complains that "the life review might be composed of selected rather than random memories intended to help the experiencer in some fashion," and that I never consider any such possibility. But this is explicitly not what the Fenwicks found, and I cannot be faulted for neglecting Serdahely's findings about a small sample of NDErs when I was addressing the Fenwicks' findings concerning a much larger sample.

Finally, Serdahely writes: "Augustine never discussed the [primarily salutary] aftereffects of NDEs... [T] he same [effects] cannot be said for those who have suffered actual hallucinations... If NDEs are truly hallucinations, then Augustine needs to reconcile this disparity." There are at least two possible explanations. First, most NDErs had their NDEs as a result of coming close to death. NonNDErs who come close to death experience similar, but less robust, transformations as NDErs (van Lommel, van Wees, Meyers, and Elfferich, 2001). This alone is sufficient to explain transformations accompanying hallucinations at the brink of death but absent from those occurring under other, non-life-threatening circumstances. Second, NDErs report more marked transformations than those who come close to death without ever experiencing an NDE. This means that NDErs report an additional transformative effect on top of that produced by coming close to death, and that additional effect requires an explanation.

And there is, in fact, a ready one: the fact that the vast majority of Western NDErs take a survivalist interpretation of their NDEs (Augustine, 2007, p. 207). Pim van Lommel and colleagues found that, between their 2-year and 8-year follow-up interviews, NDErs' belief in life after death had increased slightly, while their interest in spirituality, social attitudes, and the meaning of life increased dramatically, indicating that "this process of [salutary] change after [an] NDE tends to take several years to consolidate" (van Lommel, van 
Wees, Meyers, and Elfferich, 2001, p. 2043). This, in turn, suggests that "NDE transformations do not result from the NDE itself, but ... from the added layers of meaning and interpretation NDErs place on their NDEs" (Augustine, 2007, p. 210). In my view, a survivalist interpretation of NDEs among most Western NDErs is primarily responsible for the additional transformative effect found among NDErs.

This hypothesis is directly testable by constructing a prospective study in which NDErs are asked about how they interpret their experiences, allowing researchers to analyze the different transformative effects reported by survivalist, nonsurvivalist, and uncertain NDErs. If I am right, one would expect to see the same sorts of transformations among nonsurvivalist NDErs that have been found for nonNDErs who came close to death. Alternatively, if future research falsifies that prediction, I suspect that it will be difficult for advocates of the hallucination hypothesis to provide another plausible candidate for the source of the additional transformative effect reported by NDErs. Only time will tell.

\section{References}

Augustine, K. (2007). Does paranormal perception occur in near-death experiences? Journal of Near-Death Studies, 25, 203-236.

Crookall, R. (1972). Case-book of astral projection, 545-746. Secaucus, NJ: University Books.

Fox, O. (1962). Astral projection: A record of out-of-body experiences. New Hyde Park, NY: University Books.

Greyson, B. (1983). The Near-Death Experience Scale: Construction, reliability, and validity. Journal of Nervous and Mental Disease, 171, 269-275.

Irwin, H. J. (1985). Flight of mind: A psychological study of the out-of-body experience. Metuchen, NJ: Scarecrow Press.

Irwin, H. J. (2000). The disembodied self: An empirical study of dissociation and the outof-body experience. Journal of Parapsychology, 64, 261-276.

Twemlow, S. W., Gabbard, G. O., and Coyne, L. (1982). A multivariate method for the classification of preexisting near-death conditions. Anabiosis: The Journal of NearDeath Studies, 2, 132-139.

van Lommel, P., van Wees, R., Meyers, V., and Elfferich, I. (2001). Near-death experiences in survivors of cardiac arrest: A prospective study in the Netherlands. Lancet, 358, 2039-2045. 